\title{
Three Pathways of Colonic Carcinogenesis in Rats
}

\author{
CARLOS A. RUBIO \\ Department of Pathology, Karolinska Institute and University Hospital, Stockholm, Sweden
}

\begin{abstract}
Background: Colorectal cancer (CRC) is one of the more intensively studied human malignancies. For many years, the general view has been that the vast majority of CRCs in humans evolve from conventional (tubular or villous) adenomas via the adenoma-carcinoma pathway. More recently, serrated colorectal polyps (hyperplastic polyps, sessile serrated polyps and traditional serrated adenomas) have emerged as an alternative pathway of colorectal carcinogenesis in humans. Archival sections from early experiments in Sprague-Dawley (SD) rats injected with dimethylhydrazine $(D M H)$ were reviewed and the histology of colonic neoplasias was re-evaluated. Out of 215 colonic neoplasias, 9\% were serrated adenomas and 6\% serrated carcinomas, $11 \%$ conventional adenomas, $39 \%$ highly differentiated carcinomas, $21 \%$ gut-associated lymphoid tissue (GALT) carcinomas, $13 \%$ signet-ring cell carcinomas, and $1 \%$ villous carcinomas. In a more recent review of archived sections from DMH-treated rats with colonic GALT follicles, dysplastic crypts exhibiting asymmetrical bifurcations in GALT mucosa were found in $49 \%$ and colonic GALT carcinomas in 53\% of $276 \mathrm{DMH}$-treated rats. Histology of the 146 colonic GALT-carcinomas revealed highly differentiated carcinoma in $75 \%$, signet-ring cell carcinoma in $20 \%$, mucinous carcinomas in $3 \%$ and mixed in the remaining $2 \%$. Highly differentiated carcinomas were seen to evolve from dysplastic crypts with asymmetric bifurcations and from adenomas and signet-ring cell carcinomas, and from nondysplastic crypts having goblet cells with marked anisocytosis. It is apparent that DMH treatment in SD rats induced
\end{abstract}

This article is freely accessible online.

Correspondence to: Carlos A. Rubio, MD, Ph.D., Gastrointestinal and Liver Pathology Research Laboratory, Department of Pathology, Karolinska Institute and University Hospital, 17176, Stockholm, Sweden. Tel: +46 851774527, Fax: +46 851774524, e-mail: Carlos.Rubio@ki.se

Key Words: Colon cancer, rats, conventional carcinoma pathway, serrated carcinoma pathway, GALT carcinoma pathway, review. conventional adenomas, conventional carcinomas, serrated adenomas, serrated carcinomas and GALT carcinomas. The paradigm permits to monitor in detail the early histological steps that epitomize the three alternative pathways of colonic carcinogenesis in SD rats. This model might be useful for analyzing different molecular aberrations evolving during the conventional adenoma-carcinoma pathway, the serrated carcinoma pathway, and the GALT carcinoma pathway of colonic carcinogenesis, under standard laboratory conditions.

Colorectal cancer (CRC) is one of the more intensively studied human malignancies. For many years, the general view has been that the vast majority of CRCs in humans evolve from conventional (tubular or villous) adenomas via the adenoma-carcinoma pathway (1). More recently, serrated colorectal polyps (hyperplastic polyps, sessile serrated polyps and traditional serrated adenomas) have emerged as an alternative pathway of colorectal carcinogenesis in humans $(2,3)$. It has been estimated that about $30 \%$ of CRCs in humans progress via the serrated pathway (3).

\section{Colorectal Carcinogenesis in Rodents}

While attempting to produce amyotropic lateral sclerosis by feeding nuts of Cycas circinalis (a tropical fern from a family of Cycadaceae), Laqueur et al. accidentally found that rats developed colonic cancer (4). The same author subsequently demonstrated that the active carcinogen in these nuts was cycasin, a water-soluble $\alpha$-glucoside of methylazoxymethanol (5). This discovery lead Druckey et al. to administer a structurally similar compound, 1,2-dimethylhydrazine (DMH) (6) to induce colonic tumours in rats. DMH and its carcinogenic metabolites [azoxymethane (AOM) and naethylazoxymethanol] are the most commonly used compounds to study morphology, pathogenesis, prevention and treatment of experimentally induced colonic tumours (7-9). Such tumours are predominantly found in the colon (10-18).

In later years, a vast amount of literature on colorectal neoplasias induced by different carcinogens, by genetic engineering or by spontaneous mutations in mice and rats has been published. A recent search in PUBMED 
(14/11/2016) using the key words "induced colonic cancer in rats and mice" yielded 21,799 publications. This body of literature is a testimony to the expectation that experimental models might contribute to understanding the elusive process of colorectal carcinogenesis in humans.

\section{The Conventional Adenoma-Carcinoma Pathway of Colonic Carcinogenesis in Rodents}

Until recently, it was widely recognized that the administration of colonotropic carcinogens to rodents induced conventional (tubular or villous) adenomas that progressed to conventional carcinomas. Several histological classifications have been proposed to address the adenoma-carcinoma pathway in rodents. Working with AOM-treated rats, van Kouwen et al. classified colonic tumours into tubular, tubulovillous and villous adenomas or carcinomas (12). Dysplasia was defined according to the Vienna criteria applied to colonic neoplasias as either low- or high-grade dysplasia (19). Perse and Cerar also classified colonic tumours in DMH/AOM-treated rats into tubular, villous, or tubulovillous adenomas or carcinomas (13). Adenocarcinomas were classified into moderately differentiated (tubular, tubulovillous, or villous), poorly differentiated, mucinous, signet-ring cell, and undifferentiated. Summarizing a Consensus Report and Recommendations for the pathology of mouse models of intestinal cancer, Boivin $e t$ al. classified adenomas into tubular, villous, or tubulovillous, and adenocarcinomas into well-differentiated, moderately differentiated, or poorly differentiated (14). The histological carcinoma phenotypes were: tubular/tubulovillous/ villous carcinoma; mucinous carcinoma, signet-ring cell carcinoma (SRCC) and undifferentiated carcinoma. Ten years later, Washington et al. (15) published a Progress Report and Recommendations regarding the original article of Boivin $e t$ al. (14). Based on the new knowledge regarding the serrated pathway of colorectal carcinogenesis in humans, Washington et al. wrote: "The morphologic characteristics of serrated architecture have not been clearly defined in animal models, and the Panel agreed that none of the models reviewed developed neoplasms that were morphologically similar to human serrated intestinal neoplasms" (15). In a more recent review, Ward et al. postulated that adenomas in rodents often develop stalks and intestinal adenocarcinomas often develop de novo from flat lesions and not from adenomas (16). Adenomas were not histologically classified. On the other hand, adenocarcinomas were subdivided into scirrhous, tubular, papillary, tubular-papillary, mucinous, signet ring, solid, undifferentiated, and mixed types. Zalatnai et al. classified colonic tumours in AOM-treated rats into adenomas with severe dysplasia and adenocarcinomas (17). Finally, Mełeń-Mucha and Niewiandomska divided adenomas into three groups: adenoma with mild, moderate, and severe dysplasia and adenocarcinomas into well, moderately, poorly differentiated, and SRCCs (18). Thus, despite disparate classifications of colonic adenomas and carcinomas in carcinogen-treated rodents, the general view was that colonic carcinomas evolved via the conventional adenoma (tubular or villous) carcinoma pathway (Figure 1a and b).

\section{Two Recently Described Alternative Pathways of Colonic Carcinogenesis in Rats}

The serrated carcinoma pathway. In a recent review of archival sections from early experiments (21-28), it was found that out of the 215 colonic neoplasias evolving in Sprague-Dawley (SD) rats injected with $\mathrm{DMH}, 9 \%$ were serrated adenomas and 6\% serrated carcinomas (20). In addition, $11 \%$ were conventional adenomas, $39 \%$ highly differentiated carcinomas, $21 \%$ gut-associated lymphoid tissue (GALT) carcinomas, $13 \%$ signet-ring cell carcinomas, and $1 \%$ villous carcinomas. It is apparent that $\mathrm{DMH}$ treatment in SD rats induced conventional adenomas, conventional carcinomas, serrated adenomas, serrated carcinomas (Figure 1c and d), and GALT carcinomas (20).

The GALT carcinoma pathway. The mucosa of the colon in rats may be divided into two quantitatively different domains. One domain, built with crypts exhibiting goblet cells and columnar cells, occupies the vast majority of the colorectal mucosa. The other domain, covering tiny organized lymphoid follicles, is referred to as GALT mucosa. The epithelium of the GALT mucosa is described as lined with cuboidal cells, few or no goblet cells, and scattered "M cells" (so-called because of broad invaginations or microfolds) (28). M Cells have the capacity to absorb luminal antigens, macromolecules and microorganisms via clathrin-mediated endocytosis (28). Luminal antigens, macromolecules and microorganisms are subsequently hauled into antigen-presenting cells (macrophages, B-cells and dendritics cells) from where they are transferred to gut-indigenous, thymus-independent lymphoid tissue for immediate immunological processing (28). The constellation of M cells-lymphoid tissue builds a lympho-epithelial immunological cross-talk unit, a relay complex for gut recognition of antigens. On the other hand, the function of the GALT-free mucosal domain is to protect underlying structures, to absorb fluids, vitamins and some nutrients, and to lubricate the faeces by virtue of its production of mucus.

When colectomy specimens from untreated rats removed at autopsy were transilluminated in a translucent photography light box, two lumps were seen in the proximal colon (caecum), two in the transverse colon and two in the distal colon (Figure 2a), near the rectal border. Histology of the six colonic lumps revealed GALT follicles. Interspersed in the cuboidal epithelium, colonic crypts were found (Figure 2b). 

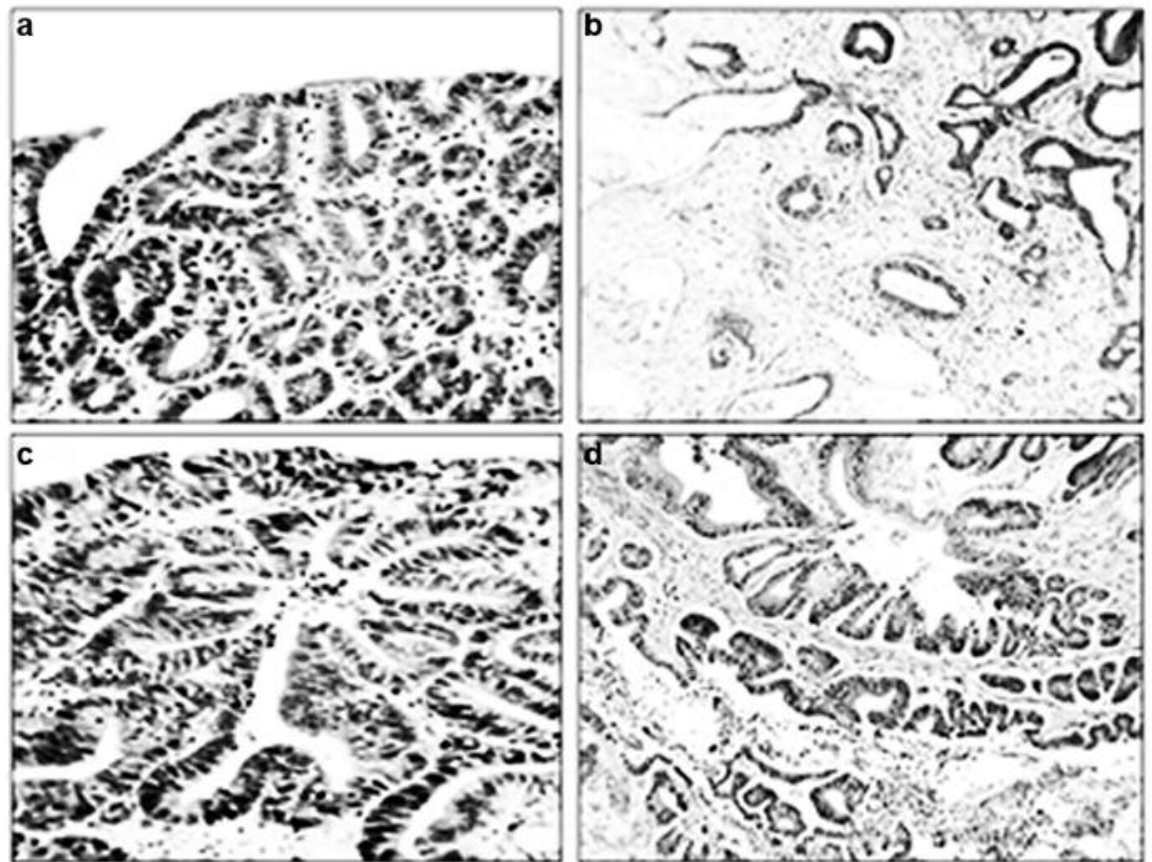

Figure 1. Exemplary tissue sections from dimethylhydrazine-treated Sprague-Dawley rats. a: Conventional tubular adenoma. Note circumscribed colonic non-invasive neoplasia built with crowded crypts lined with dysplastic cells, b: Conventional (tubular) invasive carcinoma. c: Serrated adenoma. Note unlocked serrations lined with dysplastic cells, $d$ : Serrated invasive carcinoma. Hematoxylin-eosin, $\times 10$.
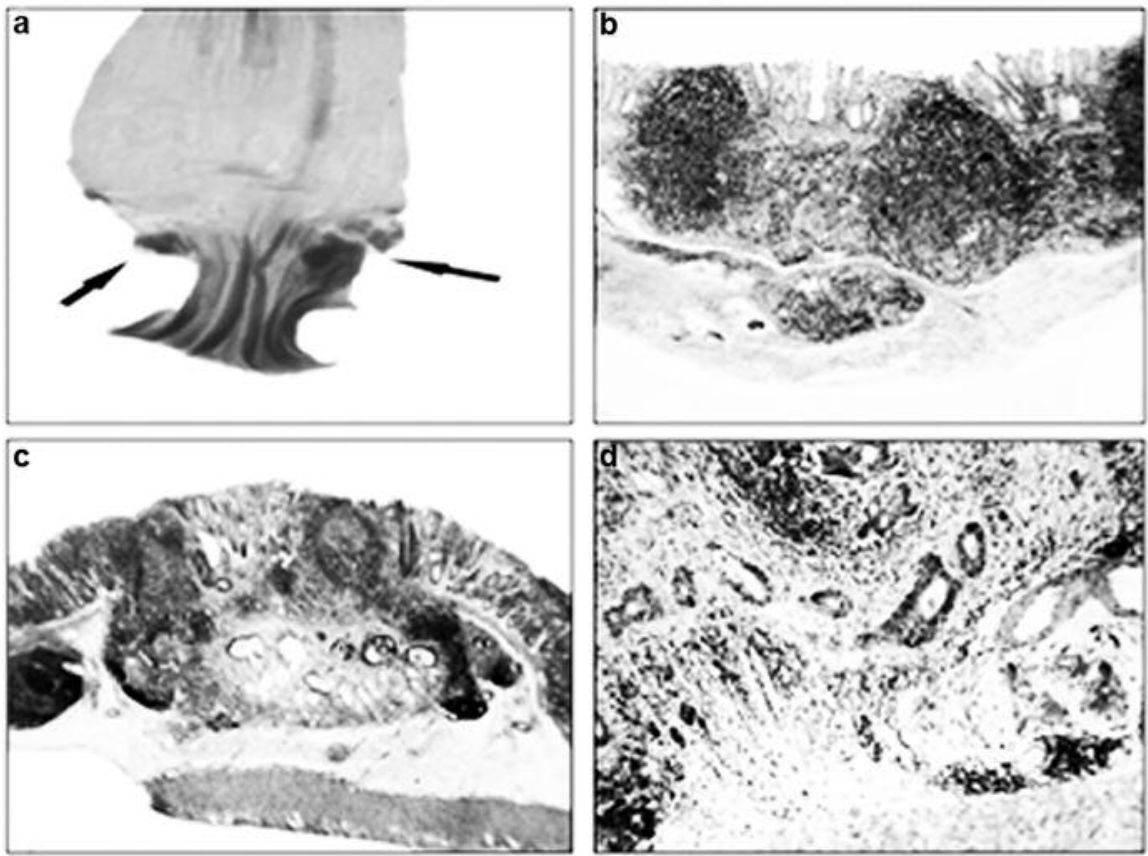

Figure 2. Exemplary tissue sections from dimethylhydrazine-treated Sprague-Dawley rats. a: Gross view of colectomy specimen subjected to transillumination. In the distal colon, near the rectum, two lumps (at arrows) can be seen. Histology showed gut-associated lymphoid tissue (GALT) mucosa. $b$ : GALT mucosa in a control rat (hematoxylin-eosin, $\times 4$ ). c: GALT carcinoma (hematoxylin-eosin, $\times 2$ ), d: Detail from a GALT carcinoma showing invasive carcinoma surrounded by lymphoid tissue (hematoxylin-eosin, $\times 10$ ). 


\section{Histological Aberrations in the GALT Mucosa of DMH-treated SD Rats}

In a previous review of archived sections from $276 \mathrm{DMH}-$ treated rats with colonic GALT follicles (29), non-dysplastic crypts were found with structural anomalies such as asymmetrical bifurcations and abnormalities in spatial orientation interspersed in the cuboidal epithelium. These non-dysplastic crypt aberrations were recorded in $20 \%$ $(n=56)$ of the 276 rats examined. The crypt epithelium was usually eosinophilic with few or no goblet cells. In addition, dysplastic crypts exhibiting asymmetrical bifurcations, or anomalies in spatial orientation in GALT mucosa were found in $49 \%(\mathrm{n}=135)$ of the 276 DMH-treated rats. Dysplastic crypts with structural aberrations were recorded in $7 \%$ of the adenomas and in 53\% of the GALT carcinomas (29). Twenty adenomas $(7 \%)$ were detected on top of the GALT mucosa in the $276 \mathrm{DMH}$-treated rats.

\section{High Frequency of Colonic GALT Carcinomas in DMH-treated Rats}

High frequency of GALT carcinomas in carcinogen-treated rats was first reported by Deasy et al. (30), and later confirmed by various authors (31-33).

In the aforementioned experiment (29), colonic GALT carcinomas were aIso found (Figure $2 \mathrm{c}$ and d) in $53 \%$ of the 276 DMH-treated rats. Histology of the 146 colonic GALT carcinomas revealed highly differentiated carcinoma (HDC) in $75 \%, \mathrm{SRCC}$ in $20 \%$, mucinous carcinoma in $3 \%$ and mixed in the remaining $2 \%$. HDCs were seen to evolve from dysplastic crypts with asymetric bifurcations and from adenomas and SRCCs, and from non-dysplastic crypts having goblet cells with marked anisocytosis. Invasive HDCs was present in $80 \%(n=16)$ of the 20 adenomas (29).

\section{Molecular Events in Colonic Crypts}

In the normal colon, the crypts are close-packed, oriented with the cryptal luminal axis perpendicular to the mucosal surface. During crypt renewal, stem cells at the crypt bottom generate amplifying daughter cells that proliferate and differentiate while migrating upwards $(34,35)$. The adenomatous polyposis coli $(A P C)$ gene normally down-regulates WNT signalling. The APC concentration is low at the crypt bottom and high at the top (the domain of differentiated cells). WNT signalling, in contrast, is high at the bottom [where stem cells reside (36)] and low at the top. Hence, WNT and APC gradients are important in crypt formation and regulation. Since both APC and WNT signalling components (e.g. survivin) are required for mitosis, this mechanism establishes a zone in the lower crypt where conditions are optimal for maximal cell division and mitosis orientation (symmetric versus asymmetric) $(34,35)$. Mutations in the APC gene are found in most CRCs, in both rodents and humans. Mutation of the APC gene causes abnormal crypt production, disorientation of the crypts, and increased crypt production leading to colorectal adenomas. Thus, the asymmetric fission of non-dysplastic crypts found in GALT follicles, most likely, is tailored by mutations in the $A P C$ gene generated by DMH. The asymmetric fission of non-dysplastic crypts strongly suggests that crypt fission occurs before the cell mutations that ultimately generate dysplastic crypts.

\section{An Animal Model Without GALT Carcinomas}

An important factor in the development of CRC in humans is the lifestyle, especially dietary habits (37). In this context, it should be mentioned that early experiments demonstrated that extracts of scorched broiled fish and meat contained highly mutagenic heterocyclic amines (38). Accordingly, pyrrolate from scorched amino acids and proteins were administered to rodents to study potential carcinogenesis (38). Takayama et al., found that the oral administration of 2-amino-6-methyldipyrido[1,2-a:3',3'-d]imidazole (GLU-1) isolated from a glutamic acid pyrrolate induced tumours in the large and small intestine, liver, ear duct and clitoral gland of F344 rats (39). In a later review of sections from an experiment by Takayama et al., in 53 colonic neoplasias evolving in 101 Fisher-344 (F-344) rats fed with GLU-1 for 24 months, it was found that $60 \%$ were tubular adenomas, $23 \%$ serrated adenomas, $2 \%$ villous adenomas, and $15 \%$ highly differentiated (tubular) carcinomas (40). GALT carcinomas were not found in GLU1-treated F-344 rats. One possible explanation for these findings might be that the chemical nature of the carcinogen administered is essential for the induction of GALT carcinomas in rats.

\section{Pathways of Colorectal Carcinogenesis in Humans}

It is generally recognized that the vast majority of CRCs in humans evolve in the GALT-free mucosal domain via the conventional adenoma (tubular or villous)-carcinoma pathway or the serrated adenoma-carcinoma pathway. Less frequently, CRCs develop in the GALT mucosal domain, via the GALT carcinoma pathway. In fact, a recent review indicates that only 21 GALT carcinomas have been reported in the literature on humans (41). The cause for the low frequency of CRC in the GALT mucosal domain in humans and the high frequency of GALT carcinomas in DMH-treated SD rats remains challenging and deserves to be further investigated.

\section{Conclusion}

In the present survey, three different pathways of colorectal carcinogenesis in DMH-treated SD rats are described. The model permits monitoring in detail the early histological 
steps that epitomize the three alternative pathways of colonic carcinogenesis in SD rats. This paradigm might be useful in analysing different molecular aberrations evolving during the conventional adenoma-carcinoma pathway, the serrated carcinoma pathway, and the GALT carcinoma pathway of colonic carcinogenesis, under the standard conditions of the laboratory.

\section{References}

1 Jackman RJ and Mayo CW: The adenoma-carcinoma sequence in cancer of the colon. Surg Gynecol Obstet 93: 327-330, 1951.

2 Jass JR: Serrated route to colorectal cancer: Back street or super highway? J Pathol 193: 283-285, 2001.

3 O'Brien MJ, Zhao Q and Yang S: Colorectal serrated pathway cancers and precursors. Histopathology 66: 49-65, 2015.

4 Laqueur GL, Mickelsen O, Whiting MG and Kurkland LT: Carcinogenic properties of nuts from Cycas circinalis 1. indigenous to Guam. J Natl Cancer Inst 3: 919-951, 1963.

5 Laqueur GL: The induction of intestinal neoplasms in rats with the glycoside cycasin and its aglycone. Virchows Arch Pathol Anat. Physiol Klin Med 340: 151-163, 1965.

6 Druckrey H, Preussmann R, Matzkies F and Ivankovic S: Selective production of intestinal cancer in rats by $1,2-$ dimethylhydrazine. Naturwissenschaften 54: 285-286, 1967

7 Rubio C A, Nylander G and Santos M: Experimental colon cancer in the absence of intestinal contents in Sprague-Dawley rats. J Natl Cancer Inst 64: 569-572, 1980.

8 Rubio CA and Nylander G: Surgical resection of the rat colon: effects on carcinogenesis by 1,2-dimethylhydrazine. J Natl Cancer Inst 68: 813-815, 1982.

9 Rubio CA, Nylander G, Wallin B, Sveander M, Alun ML and Duvander A: Carcinogenesis at colonic anastomotic sites. An animal study. Dis Colon Rectum 27: 468-470, 1984.

10 Rubio CA, Wallin B, Ware J, Sveander M and Duvander A: Effect of indomethacin in autotransplanted colonic tumors. Dis Colon Rectum 32: 488-491, 1989.

11 Shetye JD, Rubio CA, Harmenberg U, Ware J, Duvander A and Mellstedt HT: Tumor-associated antigens common to humans and chemically induced colonic tumors of the rat. Cancer Res 50: 6358-6563, 1990.

12 van Kouwen MC, Laverman P, Hanm J, Oyen WJ, Nagengast FM and Drenth JP: Noninvasive monitoring of colonic carcinogenesis: feasibility of [ $\left.{ }^{18} \mathrm{~F}\right] \mathrm{FDG}-\mathrm{PET}$ in the azoxymethane model. Nucl Med Biol 33: 245-248, 2006.

13 Perše M and Cerar A: Morphological and molecular alterations in 1,2 dimethylhydrazine and azoxymethane induced colon carcinogenesis in rats. J Biomed Biotechnol 2011: 473964, 2011.

14 Boivin GP, Washington K, Yang K. Ward JM, Pretlow TP, Russell R, Besselsen DG, Godfrey VL, Doetschman T, Dove WF, Pitot HC, Halberg RB, Itzkowitz SH, Groden J and Coffey RJ: Pathology of mouse models of intestinal cancer: consensus report and recommendations. Gastroenterology 124: 762-777, 2003.

15 Washington MK, Powell AE, Sullivan R. Sundberg JP, Wright N, Coffey RJ and Dove WF: Pathology of rodent models of intestinal cancer: progress report and recommendations. Gastroenterology 144: 705-717, 2013.
16 Ward JM and Treuting PM: Rodent intestinal epithelial carcinogenesis: pathology and preclinical models. Toxicol Pathol 42: 148-161, 2014

17 Zalatnai A, Lapis K, Szende B, Rásó E, Telekes A, Resetár A and Hidvégi M: Wheat germ extract inhibits experimental colon carcinogenesis in F-344 rats. Carcinogenesis 22: 1649- 1652, 2001.

18 Mełen-Mucha G and Niewiadomska H: Frequency of proliferation, apoptosis, and their ratio during rat colon carcinogenesis and their characteristic pattern in the dimethylhydrazine-induced colon adenoma and carcinoma. Cancer Invest 20: 700-712, 2002.

19 Rubio CA, Nesi G, Messerini L, Zampi GC, Mandai K, Itabashi $\mathrm{M}$ and Takubo K: The Vienna classification applied to colorectal adenomas. J Gastroenterol Hepatol 21: 1697-1703, 2006.

20 Rubio CA: Traditional serrated adenomas and serrated carcinomas in carcinogen-treated rats. J Clin Patholdoi: 10.1136/jclinpath-2016-204037, 2016.

21 Rubio CA, Nylander G, Wahlin B. Sveander M, Duvander A and Alun ML: Monitoring the histogenesis of colonic tumors in the Sprague-Dawley rat. J Surg Oncol 3: 225-228, 1986.

22 Rubio CA, Nylander G., Sveander, Duvander A and Alun ML: Minimal invasive carcinoma of the colon in rats. Am J Pathol 123: 161-165, 1986.

23 Rubio CA, Wallin B, Sveander M and Duvander A: A model for the study of metastases from colonic tumors by autotransplantation. Dis Colon Rectum 30: 884-887, 1987.

24 Rubio CA: Autografting colonic adenocarcinomas into the colonic submucosa in rats. In Vivo 1: 65-68,1987.

25 Shetye J and Rubio CA: The chronological appearance of flat colonic neoplasias in rats. In Vivo 18: 197-202, 2004

26 Rubio CA: Apoptotic differences in experimentally induced colorectal rat tumours. Apoptosis 3: 35-39, 1998.

27 Rubio CA and Rivera F: Quantification of acid mucins in the descending colon of rats having simultaneously growing colonic tumors. APMIS 99: 993-996, 1991.

28 Neutra M, Mantis N and Kraehenbuhl J P: Collaboration of epithelial cells with organized mucosal lymphoid tissues. Nature Immunol 2: 1004-1009, 2001.

29 Rubio CA: The third pathway of colon carcinogenesis in carcinogen-treated rats. J Clin Pathol 2016 (in press).

30 Deasy JM, Steele G Jr, Ross DS, Lahey SJ, Wilson RE and Madara J: Gut-associated lymphoid tissue and dimethylhydrazine-induced colorectal carcinoma in the Wistar/Furth rat. J Surg Oncol 124: 36-40, 1983.

31 Martin MS, Hammann A and Martin F: Gut-associated lymphoid tissue and 1,2-dimethylhydrazine intestinal tumors in the rat: a histological and immunoenzymatic study Int J Cancer 38: 75-80, 1986.

32 Rubio CA: Lymphoid tissue-associated colonic adenocarcinomas in rats. In Vivo 1: 61-64, 1987.

33 Rubio CA, Shetye J and Jaramillo E: Non-polypoid adenomas of the colon are associated with subjacent lymphoid nodules. An experimental study in rats. Scand J Gastroenterol 34: 504-508, 1999.

34 Tan CW, Hirokawa Y, Gardiner BS, Smith DW and Burgess AW: Colon cryptogenesis: asymmetric budding. PLoS One 8: e78519, 2013.

35 Boman BM and JZ and Fields: An APC:WNT counter-currentlike mechanism regulates cell division along the human colonic crypt axis: a mechanism that explains how $A P C$ mutations induce proliferative abnormalities that drive colon cancer development. Front Oncol 3: 244 250, 2013. 
36 Rubio CA: Putative stem cells in mucosas of the esophagogastrointestinal tract. Chapter 10. In: Stem Cells, Regenerative Medicine and Cancer. Singh SR (ed.). Haupauge, NY, USA: Nova Science Publishers, Inc. pp. 279-308, 2011.

37 Chen Z, Wang PP, Woodrow J, Zhu Y, Roebothan B, Mclaughlin JR and Parfrey PS: Dietary patterns and colorectal cancer: results from a Canadian population-based study. Nutr J 14: 8-14, 2015.

38 Masuda $\mathrm{M}$ and Takayama S: Intestinal tumors in rats induced by mutagens from glutamic acid pyrolysate. Exp Pathol 26: 123129,1984

39 Takayama S, Masuda M, Mogami M, Ohgaki H, Sato S and Sugimura T: Induction of cancers in the intestine, liver and various other organs of rats by feeding mutagens from glutamic acid pyrolysate. Gan 75: 207-213, 1984.
40 Rubio CA and Takayama S: Difference in histology and size in colonic tumors of rats receiving two different carcinogens. J Environ Pathol Toxicol Oncol 13: 191-197, 1994.

41 Rubio CA and Schmidt PT: Gut-associated lymphoid tissue (GALT) Carcinoma or dome carcinoma? Anticancer Res 36: 5385-5387, 2016.
Received November 15, 2016

Revised December 2, 2016

Accepted December 6, 2016 